\title{
Rabdomiólise induzida pelo exercício: biomarcadores, mecanismos fisiopatológicos e possibilidades terapêuticas
}

\author{
Exercise-induced rhabdomyolysis: biomarkers, pathophysiological \\ mechanisms and therapeutic possibilities
}

Gustavo C. Lopes*

Luciane P. da Costa

\section{Resumo}

O termo "rabdomiólise" significa literalmente quebra ou ruptura do tecido muscular esquelético, que é derivado de uma de suas principais características: a deterioração deste tecido. A rabdomiólise possui uma etiologia bastante variada sendo conhecida há muito tempo. A interação com vários fármacos é bem-descrita pela literatura. Episódios curtos de exercício agudo ou severo também podem aumentar em muito a probabilidade de seu aparecimento. Além disso, estudos recentes mostram que sob certas circunstâncias esta interação também pode ser observada com a utilização de alguns tipos de suplementos alimentares, um comportamento comum reportado por praticantes de atividade física em geral. Esta síndrome normalmente está associada com uma lesão celular maciça, que pode levar à dor e eventual e potencialmente a óbito. Os mecanismos fisiopatológicos que originam a rabdomiólise são relativamente bem-descritos, porém diversos estudos recentes sugerem

Revista HUPE, Rio de Janeiro, 2013;12(4):59-65 doi:10.12957/rhupe.2013.8713 que alterações significativas no equilíbrio antioxidante do organismo estão associadas com esta síndrome. Contudo, a utilização de antioxidantes como estratégia terapêutica ainda apresenta resultados controversos. Por outro lado, trabalhos recentes mostram que o treinamento adequado exerce um efeito protetor contra o aparecimento de danos celulares induzidos pelo exercício, promovidos pela manutenção do estado antioxidante. O conhecimento insatisfatório apresentado por professores de educação física sobre esta síndrome merece atenção, pois estes profissionais trabalham atendendo diariamente a centenas de pessoas. Este quadro se torna ainda mais problemático quando uma parte significativa da população começa a participar de regimes de atividade física em condições não controladas. Sendo assim, é de fundamental importância o reconhecimento desta síndrome na prática desportiva de modo que possa ser realizada uma intervenção precoce. Futuros estudos são necessários para o diagnóstico da

*Endereço para correspondência: Laboratório de Atividade Física e Promoção da Saúde, IEFD, UERJ. Rua São Francisco Xavier, 524, bloco F, $8^{\circ}$ andar, sala 8.121 Rio de Janeiro, RJ, Brasil. CEP: 20550-900. E-mail: gustavo.casimiro@gmail.com 
rabdomiólise, facilitando o monitoramento e prevenção de riscos no exercício físico. Em nosso laboratório estamos avaliando parâmetros bioquímicos que poderiam estar associados com possíveis mecanismos nessa síndrome.

Descritores: Rabdomiólise; Dano celular; Redox; Exercício físico.

\section{Abstract}

The term "rhabdomyolysis" means literally break or rupture of skeletal muscle tissue, its name is derived from one of its main characteristics which is the deterioration of this tissue. The rhabdomyolysis possesses a varied etiology and has been known for a long time. The interaction with many drugs is well described in the literature. Brief episodes of acute and/or severe exercise can also raise the probability of this syndrome. Besides that, recent studies shows which under certain circumstances this interaction can be observed with dietary supplements, utilized in large scale for physical practitioners in general. This syndrome is normally associated with massive cell injury which can trigger pain and eventually lead to death in some cases. The pathophysiological mechanisms involved are relatively well described; however many recent studies also suggest that alterations in redox homeostasis are associated with this syndrome. However, the usage of antioxidants as a therapeutic strategy still presents controversial results. On the other hand, recent work has shown that adequate training exerts protective effects against exercise-induced cell damage promoted by the maintenance of the antioxidant state. The poor knowledge presented by Physical Education teachers deserves attention because these professionals work attending hundreds of people on a daily basis. This feature is even more problematic when a significant part of the population takes part in physical activity regimens in non-controlled conditions. So, it is necessary to recognize this syndrome in sports practice in a way that allows early intervention. Future studies are necessary for the rhabdomyolysis diagnosis, making it easier to prevent and monitor risks in physical exercise. In our lab we are evaluating biochemical parameters which could be associated with possible mechanisms influencing in this syndrome.

Keywords: Rhabdomyolysis; Cell damage; Redox; Physical exercise.

\section{Introdução}

O nome rabdomiólise vem da palavra rabdomio, que significa músculo esquelético, e lise, que significa quebra ou ruptura. Esta síndrome é caracterizada principalmente por danos na musculatura esquelética resultando em extravasamento do conteúdo celular, como: mioglobina, potássio, fosfato, enzimas, entre outros. Seus primeiros sinais e sintomas podem incluir mialgias, fraqueza muscular e o escurecimento da urina. ${ }^{1}$

Sua identificação inicial se deu após a II Guerra Mundial em 1941, em um evento denominado The blitz of London. Nesse episódio diversas casas foram bombardeadas, gerando muitas mortes por desabamento. Características semelhantes também foram observadas em diversos soldados, queimados em trincheiras, que posteriormente levariam a sua identificação naqueles que sobreviveram. ${ }^{2}$ Além do traumatismo corporal, existem muitas outras situações que podem aumentar de forma substancial o risco de aparecimento da rabdomiólise, como convulsões, ingestão de álcool, infecções, cirurgias, hipotireoidismo e o uso de certas drogas como cocaína e estatinas. ${ }^{3}$

O diagnóstico da rabdomiólise se baseia, em grande parte, na presença de elevados níveis plasmáticos da enzima creatina quinase (CK; EC 2.7.3.2), cujos valores até 170 UI/l são geralmente considerados normais. Considera-se risco quando a concentração de CK ultrapassa a faixa de $500 \mathrm{UI} / \mathrm{l}$, carecendo de exames complementares. Valores maiores do que 5.000 UI/l estão associados com um maior risco para 
insuficiência renal. ${ }^{3,4}$

Outras enzimas também podem estar presentes como transaminase glutâmicopirúvica (TGP; EC 2.6.1.1), transaminase oxaloacética (TGO; EC 2.6.1.2) e lactato desidrogenase (LDH; EC 1.1.1.2.7). ${ }^{1,3,4}$ Sua quantificação é muito empregada em diagnósticos clínicos, pois pode indicar a gravidade da lesão e origem do tecido lesionado.

A prática regular de exercícios físicos apresenta inúmeros benefícios para a saúde, fato que justifica sua utilização como terapêutica complementar em diversas doenças. ${ }^{5}$ Porém, há muito já se sabe que o exercício físico é uma forma de estresse fisiológico, dando origem à síndrome de adaptação geral, que é dividida em: 1) estresse; 2) alarme; 3) adaptação e 4) platô. ${ }^{6}$ Em situações onde a carga de treinamento se encontra bem-distribuída e com intensidade suficiente, o exercício gera uma fase inicial que mimetiza uma reação inflamatória em resposta ao dano tecidual e produção de espécies reativas de oxigênio (EROs). Sendo assim, caso ocorra um desequilíbrio entre a relação estímulorepouso, como durante a prática excessiva de exercícios extenuantes ou durante sessões agudas, o organismo pode apresentar riscos à saúde. ${ }^{7}$

O grau de condicionamento físico parece exercer um efeito protetor importante contra o surgimento da rabdomiólise (exertional rhabdomyolysis). É citado por alguns autores para descrever um quadro normalmente não letal em resposta ao exercício extenuante em indivíduos treinados. Por outro lado, em indivíduos sedentários que se submetem a testes físicos para avaliação de aptidão física, o risco de evolução dos parâmetros clínicos é ainda maior e, não raro, pode ser fatal. ${ }^{8}$

Outro componente que gera grande preocupação é a interação fármaco-exercício. Diversos relatos são descritos em indivíduos praticantes de exercícios, casos nos quais existe a associação entre o aparecimento de rabdomiólise com o uso de suplementos alimentares ${ }^{9}$ e com esteroides anabolizantes androgênicos. ${ }^{10}$ Além disso, a utilização de estatinas em portadores de dislipidemia parece apresentar um grande risco para o aparecimento da rabdomiólise. ${ }^{11}$

Outro grupo bastante suscetível é o dos militares, já que parte de sua rotina de treinamento é constituída por longas caminhadas (marchas de $50-80 \mathrm{~km}$ ) e testes físicos podendo levar à rabdomiólise. ${ }^{12}$ Hooda \& Narula observaram um caso não fatal de rabdomiólise que levou à insuficiência renal aguda em um oficial após uma corrida de 16 $\mathrm{km} .{ }^{13}$ Por outro lado, Kuklo e colaboradores relataram a ocorrência de um caso fatal de rabdomiólise em um soldado após uma corrida de apenas $3.200 \mathrm{~m}$. No momento que deu entrada na emergência apresentava hipertermia e taquicardia. Seus valores de CK chegaram a $800.000 \mathrm{UI} / 1$ (valores de referência aproximados: < $150 \mathrm{UI} / \mathrm{l})$. Setenta e duas horas após dar entrada no hospital e apesar de todas as tentativas de tratamento o quadro se agravou, levando-o à morte nas 24 horas subsequentes. ${ }^{14}$

Estes relatos indicam que diversos fatores, além do estado físico, podem participar da etiologia da rabdomiólise e devem ser, portanto, considerados em indivíduos que almejam se exercitar ou participar de algum tipo de prova física.

\section{Mecanismos fisiopatológicos}

O mecanismo de extravasamento das enzimas indicadoras de dano tecidual (CK, LDH, TGO, TGP e GGT) parece estar relacionado com a depleção dos estoques intracelulares de glutationa (GSH). O GSH é um tripeptídeo formado pelos aminoácidos: glicina, ácido glutâmico e cisteína, sendo este último o responsável pela maior parte de suas propriedades bioquímicas. Seu principal sítio de produção é o fígado, enquanto que seus valores plasmáticos são mantidos estáveis para atender às necessidades dos órgãos periféricos. ${ }^{15}$

Diversos fatores estão relacionados com a depleção de GSH, inclusive o exercício (Figura 1), o qual, por ser capaz de produzir espécies reativas de oxigênio (EROs), colabora com o 
extravasamento destas enzimas para o plasma pelo mecanismo de peroxidação lipídica das membranas celulares. ${ }^{15}$

O exercício agudo/excessivo pode levar também ao aparecimento de mioglobina no plasma, em resposta ao dano tecidual. O grupo heme da mioglobina plasmática, em estado férrico $\left(\mathrm{Fe}^{3+}\right)$, ao alcançar o rim, a $\mathrm{Mb}^{3+}$ passa por um ciclo redox atingindo o estado ferril $\left(\left[\mathrm{Fe}^{4+}=\mathrm{O}\right]^{2+}\right)$ que possui afinidade e capacidade de gerar oxidação lipídica do epitélio renal. ${ }^{16}$ Esta reação leva à formação de $\mathrm{F}_{2}$-isoprostanos, um derivado tóxico gerado pela mobilização de ácido araquidônico dos fosfolipídeos de membrana com capacidade vasoconstritora. ${ }^{17}$ Por sua vez, essa vasoconstrição gera um ciclo vicioso, pois a isquemia causada diminui o pH sanguíneo (Figura 2). A reatividade da mioglobina é fortemente aumentada em valores de $\mathrm{pH}$ menores do que $7,0,{ }^{18}$ favorecendo a insuficiência renal aguda. ${ }^{16}$

A abordagem terapêutica básica envolve basicamente a prevenção e o tratamento da insuficiência renal e em muitos casos se utiliza uma hidratação precoce e vigorosa associada ou não a uma diurese alcalina forçada. ${ }^{1,3}$ Estudos experimentais mostraram que o tratamento com bicarbonato de potássio aumentou o clearance de creatinina (um indicador de melhora da função renal) e diminuiu a formação de $\mathrm{F}_{2}$-isoprostanos urinários. ${ }^{16}$

A desidratação também é um fator de risco, pois diminui o fluxo tubular e aumenta a reabsorção de água favorecendo a vasoconstrição renal. O redirecionamento do fluxo sanguíneo para a pele ocorre para dissipar o calor, mas gera isquemia gastrointestinal liberando endotoxinas na corrente sanguínea, quadro esse conhecido como endotoxemia. A liberação de endotoxinas estimula a produção de citocinas que amplificam a reação inflamatória e provocam instabilidade hemodinâmica, ${ }^{19}$ favorecendo o surgimento de uma nova complicação denominada síndrome compartimental, complicação que se desenvolve

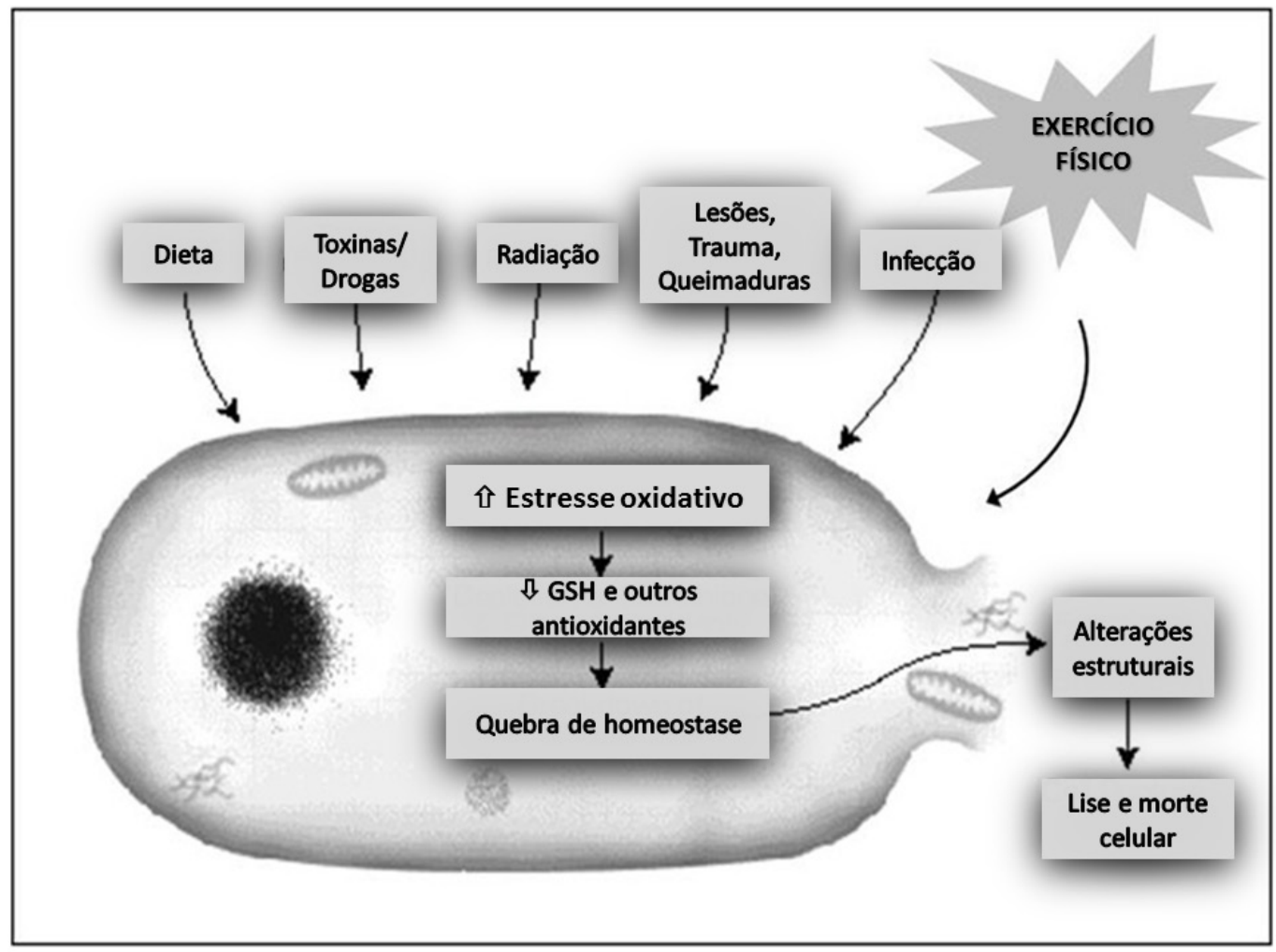

Figura 1. Fatores envolvidos na lise celular que possivelmente podem levar ao extravasamento de enzimas para o plasma.

Fonte: Adaptado de Glutathione: Systemic protectant against oxidative and free radical damage. ${ }^{15}$ 


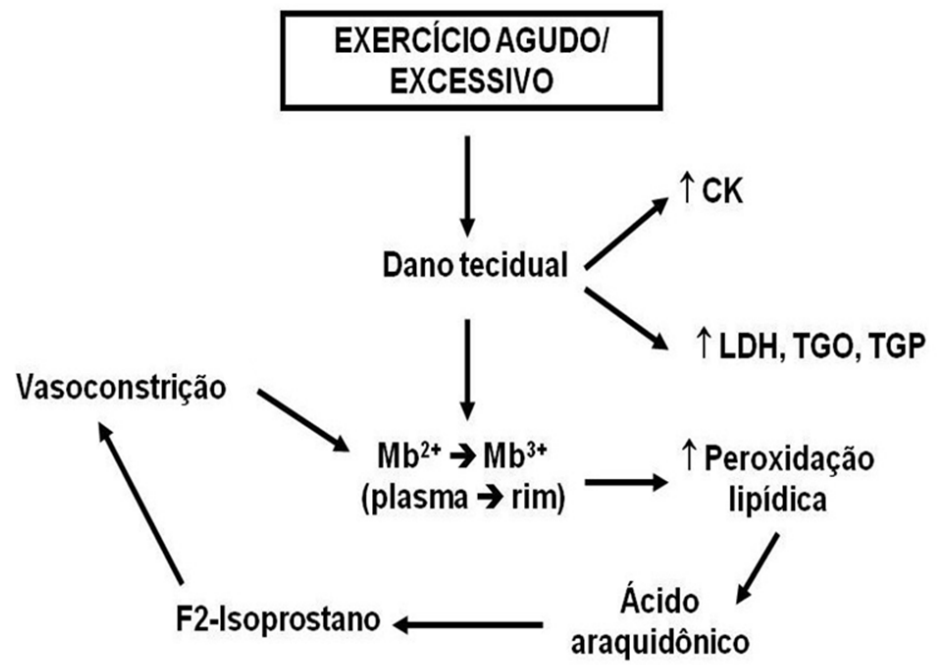

Figura 2. Mecanismo fisiopatológico da rabdomiólise e sua relação com a insuficiência renal.

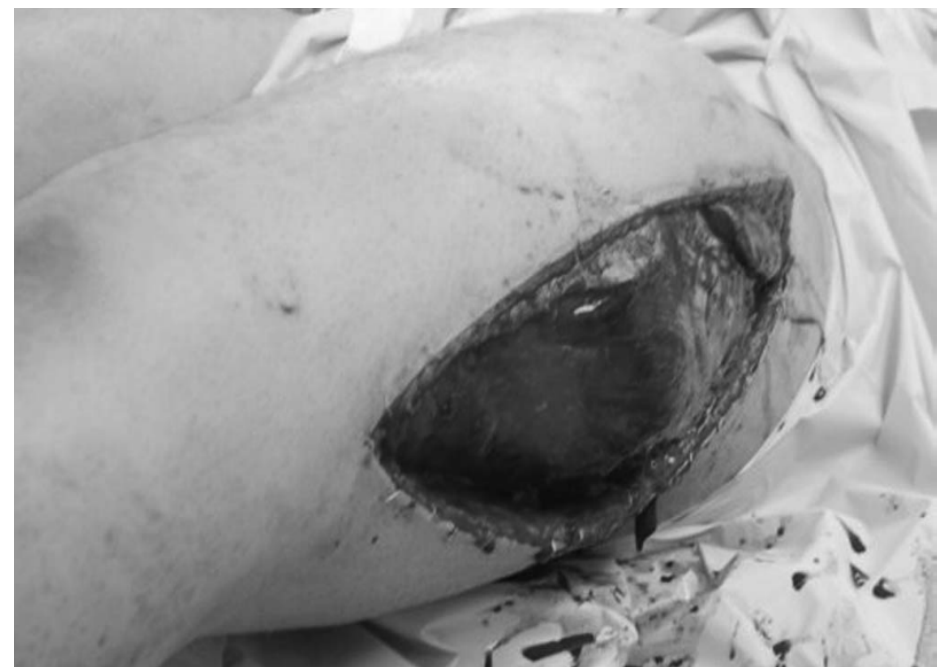

Figura 3. Indivíduo após a realização de uma fasciotomia na face lateral da coxa esquerda. ${ }^{9}$

no músculo esquelético quando sua perfusão não é adequada em virtude dos eventos descritos anteriormente.

A exposição prolongada pode provocar paralisia nervosa irreversível, com valores de pressão compartimental maiores que 30 mmHg. ${ }^{20}$ Neste caso, torna-se necessária a intervenção cirúrgica para descompressão parcial (fasciotomia) ou total (fasciectomia), a fim de diminuir a pressão no local afetado (Figura 3). ${ }^{11}$

Recentemente Margonis e colaboradores ${ }^{21}$ observaram que indivíduos que participaram de um protocolo de treinamento de seis vezes por semana, com $80 \%$ RM na faixa de seis a oito repetições apresentaram um quadro de estresse oxidativo. ${ }^{21}$ Além disso, neste mesmo grupo foram observados aumentos significativos de CK no plasma, fato que sugere uma possível relação desta condição com o aparecimento de rabdomiólise. ${ }^{19} \mathrm{~A}$ adequação dos parâmetros de carga do exercício consiste em uma alternativa bastante promissora. Para algumas categorias de indivíduos, porém, este artifício pode se tornar inviável, devido a fatores externos, como cronogramas de competições (atletas em geral) ou obrigações institucionais (militares).

A depleção dos estoques de antioxidantes endógenos sugere que o estado redox pode estar relacionado com a lesão celular, gerando o extravasamento de enzimas para o plasma. Paralelamente, o envolvimento das EROs nos 
mecanismos fisiopatológicos da rabdomiólise relacionados com o comprometimento da função renal levanta a hipótese de que a utilização de antioxidantes possa ser uma ferramenta terapêutica promissora. ${ }^{21}$ Por outro lado, a utilização de antioxidantes em atletas e indivíduos fisicamente ativos é uma prática realizada por muitas pessoas no intuito de buscar uma melhora no desempenho, fato que ainda apresenta resultados controversos.

Estudos realizados em nosso laboratório observaram que militares treinados não apresentaram quaisquer alterações deletérias no estado redox após uma corrida de 3.000 metros, sugerindo que o estado físico pode realmente ser um efeito protetor contra o aparecimento de rabdomiólise. ${ }^{28}$ Parte desse efeito provavelmente está relacionada com a aplicação de programas de treinamento adequados, além da adoção de hábitos saudáveis de alimentação incluindo fontes dietéticas de antioxidantes. ${ }^{23} \mathrm{O}$ aumento da eficiência do sistema antioxidante em atletas mostra um efeito benéfico do exercício e também deve ser considerado. ${ }^{24}$ Por sua vez, Brooks e colaboradores ${ }^{24}$ observaram em camundongos adequadamente treinados uma menor produção de EROs devido a uma maior capacidade de varredura ou de produção do organismo.

\section{Conclusões}

A identificação dos fatores que levam ao aparecimento desta síndrome ainda não está bem-esclarecida, porém estudos vêm sendo realizados de forma crescente. Trabalhos recentes realizados em seres humanos utilizando técnicas de biologia molecular avaliaram a presença de polimorfismos específicos, fato que poderia explicar porque existe uma grande variabilidade na resposta a um mesmo protocolo de exercício podendo levar ou não à rabdomiólise. ${ }^{8,4,9}$

Clarkson e colaboradores ${ }^{25}$ observaram que indivíduos homozigotos para o alelo MLCK (cadeia leve de miosina) do tipo $49 \mathrm{~T}$ apresentaram maiores incrementos nas concentrações plasmáticas de CK e mioglobina, em resposta ao exercício excêntrico. Por outro lado, sujeitos heterozigotos para o alelo MLCK 3788A mostraram aumentos apenas para CK, sugerindo que portadores destes genótipos apresentam um maior risco para desenvolver rabdomiólise. ${ }^{26}$ Outros estudos vêm sendo realizados na busca de genótipos que possam ajudar a esclarecer a origem destas alterações. ${ }^{26}$

Apesar de tudo o que foi demonstrado até aqui, pouco progresso tem sido feito na prevenção desta síndrome. A grande quantidade de estudos de casos publicados ${ }^{8,9,11,15}$ indica que a monitoração deste quadro é bastante limitada, só podendo ser realizada quando de fato já existem sinais clínicos evidentes. Além disso, a população que pode potencialmente ser acometida se torna bastante ampla (indivíduos saudáveis ou não, jovens ou idosos, sedentários, entre outros) quando se considera a prática de atividade física em geral. Fato que não considera apenas atletas de alto rendimento. Montero e colaboradores ${ }^{27}$ relataram em um estudo retrospectivo que indivíduos fisicamente ativos praticantes de ciclismo indoor (spinning) apresentaram esta síndrome. ${ }^{28}$

Sendo assim, é de fundamental importância o reconhecimento desta síndrome na prática desportiva, de modo que possa ser tratada mais precocemente. Futuros estudos são necessários para o diagnóstico precoce de rabdomiólise, facilitando o monitoramento e prevenção de riscos ao exercício. Estudos estão sendo conduzidos em nosso laboratório para melhor entender os mecanismos envolvidos neste processo.

\section{Referências}

1. Criddle LM. Rhabdomyolysis: Pathophysiology, recognition, and management. Crit Care Nurse. 2003;23(6):14-32.

2. Bywaters EGL, Beall D. Crush injuries with impairment of renal function. Br Med J. 1941;1(4185):427-32.

3. Huerta-Alardín AL, Varon J, Marik PE. Bench-to-bedside review: Rhabdomyolysis - an overview for clinicians. Crit Care. 


\section{5;9(2):158-69.}

4. Skenderi KP, Kavouras CA, Anastasiou N, Yiannakouris N, Matalas AL. Exertional rhabdomyolysis during a 246-km continuous running race. Med Sci Sports Exerc. 2006;38(6):1054-7.

5. American College of Sports Medicine Position Stand: Exercise for patients with coronary artery disease. Med Sci Sports Exerc. 1994;26(3):1-5.

6. Selye H. The general-adaptation-syndrome. Annu Rev Med. 1951;2:327-42.

7. Fehrenbach E, Schneider ME. Traumainduced systemic inflammatory response versus exercise-induced immunomodulatory effects. Sports Med. 2006;36(5):373-84.

8. Uchoa RB, Fernandes CR. Rabdomiólise induzida por exercício e risco de hipertermia maligna. Relato de caso. Rev Bras Anestesiol. 2003;53(1):63-8.

9. Do KD, Bellabarba C, Bhananker SM. Exertional rhabdomyolysis in a bodybuilder following overexertion: a possible link to creatine overconsumption. Clin J Sport Med. 2007;17(1):78-9.

10. Farkash U, Shabshin N, Pritsch-Perry M. Rhabdomyolysis of the deltoid muscle in a bodybuilder using anabolic-androgenic steroids: a case report. J Athl Train. 2009;44(1):98-100.

11. Tomlinson SS, Mangione KK. Potential adverse effects of statins on muscle. Phys Ther. 2005;85(5):459-65.

12. Chevion S, Moran DS, Heled Y, Shani Y, Regev G, Abbou B. Plasma antioxidant status and cell injury after severe physical exercise. Proc Natl Acad Sci USA. 2003;100(9):5119-23.

13. Hooda AK, Narula AS. Exertional rhabdomyolysis causing acute renal failure. Medical J Armed Forces India. 2005;61(4):3956.

14. Kuklo TR, Tis JE, Moores LK, Schaefer RA. Fatal rhabdomyolysis with bilateral gluteal, thigh, and leg compartment syndrome after the Army Physical Fitness Test: A case report. Am J Sports Med. 2000;28(1):112-6.

15. Kidd PM. Glutathione: Systemic protectant against oxidative and free radical damage. Altern Med Rev. 1997;2(3)155-76.

16. Moore KP, Holt SG, Patel RP, Svistunenko DA, Zackert W, Goodier D. A causative role for redox cycling of myoglobin and its inhibition by alkalinization in the pathogenesis and treatment of rhabdomyolysis-induced renal failure. J Biol Chem. 1998;273(48):31731-7.
17. Cooper CE, Vollaard NBJ, Choueiri T, Wilson MT. Exercise, free radicals and oxidative stress. Biochem Soc Trans. 2002;30(2):280-5.

18. Reeder B, Wilson MT. The effects of $\mathrm{pH}$ on the mechanism of hydrogen peroxide and lipid hydroperoxide consumption by myoglobin: A role for the protonated ferryl species. Free Radic Biol Med. 2001;30(11):1311-8.

19. Gomez-Cabrera MC, Martínez A, Santangelo G, Pallardó FV, Sastre J, Viña J. Oxidative stress in marathon runners: interest of antioxidant supplementation. Br J Nutr. 2006;96(Suppl.1):S31-3.

20. Vanholder R, Sever MS, Erek E, Lameire N. Rhabdomyolysis. J Am Soc Nephrol. 2000;11(8):1553-61.

21. Margonis K, Fatouros IG, Jamurtas AZ, Nikolaidis MG, Douroudos I, Chatznikolaou A, et al. Oxidative stress biomarkers responses to physical overtraining: Implications for diagnosis. Free Radic Biol Med. 2007;43(6):901-10.

22. Williams MH. Nutritional supplements for strength trained athletes. Sports Sci Exch. 1993;6(6):1-6.

23. Koury JC, Oliveira CF, Portella ES, Oliveira AV Jr, Donangelo CM. Effect of the period of resting in elite judo athletes: hematological indices and copper/zinc-dependent antioxidant capacity. Biol Trace Elem Res. 2005;107(3):201-11.

24. Brooks SV, Vasilaki A, Larkin LM, McArdle A, Jackson MJ. Repeated bouts of aerobic exercise lead to reductions in skeletal muscle free radical generation and nuclear factor $\kappa \mathrm{B}$ activation. J Physiol. 2008;586(16):3979-90.

25. Clarkson PM, Kearns AK, Rouzier P, Rubin R, Thompson PD. Serum creatine kinase levels and renal function measures in exertional muscle damage. Med Sci Sports Exer. 2006;38(4):623-7.

26. Yamin C, Duarte JAR, Oliveira JMF, Amir O, Sagiv M, Eynon N. IL6 (174) and TNFA (-308) promoter polymorphisms are associated with systemic creatine kinase response to eccentric exercise. Eur J Appl Physiol. 2008;104(3):57986.

27. Montero J, Lovesio C, Godoy MV, Ruiz G. Rabdomiólisis por spinning en nueve pacientes. Medicina (B. Aires). 2009;69(1):1536.

28. Santanna M, Boaventura G, Ramos DM, Casimiro-Lopes G, Sorenson M, Salerno VP. Provas de 3.000 metros aumentam a atividade de transaminases no plasma em atletas militares. At: $13^{\circ}$ Simpósio de Atividades Físicas do Rio de Janeiro; 2009; Rio de Janeiro, Brasil. 


\section{Autores}

\section{Ada Fernanda P. S. Lima}

Laboratório de Atividade Física e Promoção da Saúde. Instituto de Educação Física e Desportos. Universidade do Estado do Rio de Janeiro. Rio de Janeiro, RJ, Brasil.

\section{Alexandre H. Okano}

Departamento de Educação Física. Centro de Ciências da Saúde. Universidade Federal do Rio Grande do Norte. Natal, RN, Brasil.

\section{Alice R. Sampaio}

Laboratório de Atividade Física e Promoção da Saúde. Instituto de Educação Física e Desportos. Universidade do Estado do Rio de Janeiro. Rio de Janeiro, RJ, Brasil.

\section{Ana Paula M. Guttierres}

Laboratório de Atividade Física e Promoção da Saúde. Instituto de Educação Física e Desportos. Universidade do Estado do Rio de Janeiro. Rio de Janeiro, RJ, Brasil.

\section{Astrogildo V. Oliveira Júnior}

Departamento de Educação Física e Folclore. Colégio Pedro II. Rio de Janeiro, RJ, Brasil.

\section{Brenno S. Silva}

Programa de Pós-graduação em Ciências da Atividade Física. Universidade Salgado de Oliveira. Niterói, RJ, Brasil.

\section{Daniel A. Bottino}

Laboratório de Pesquisas Clínicas e Experimentais em Biologia Vascular. Centro Biomédico.

Universidade do Estado do Rio de Janeiro. Rio de Janeiro, RJ, Brasil.

\section{Dionizio Mendes Ramos Filho}

Laboratório de Bioenergética e Fisiologia Mitocondrial. Centro de Ciências da Saúde. Universidade Federal do Rio de Janeiro. Rio de Janeiro, RJ, Brasil.

\section{Elirez B. Silva}

Laboratório de Pesquisa Clínica Escola. Departamento de Fisioterapia. Universidade Gama Filho. Rio de Janeiro, RJ, Brasil.

\section{Ercole C. Rubini}

Laboratório de Fisiologia do Exercício. Universidade Estácio de Sá. Rio de Janeiro, RJ, Brasil.

\section{Fabrício V. A. Vasconcellos}

Centro de Investigação, Formação, Inovação, Intervenção e Desporto. Faculdade de Desporto. Universidade do Porto. Porto, Portugal.

\section{Felipe A. da Cunha}

Programa de Pós-graduação em Ciências Médicas. Universidade do Estado do Rio de Janeiro. Rio de Janeiro, RJ, Brasil.

\section{Fernanda Monteiro}

Laboratório de Atividade Física e Promoção da Saúde. Instituto de Educação Física e Desportos. Universidade do Estado do Rio de Janeiro. Rio de Janeiro, RJ, Brasil.

\section{Flávia Porto}

Programa de Pós-graduação em Ciências do Exercício e do Esporte. Universidade Gama Filho. Rio de Janeiro, RJ, Brasil. 


\section{Gustavo C. Lopes}

Laboratório de Atividade Física e Promoção da Saúde. Instituto de Educação Física e Desportos. Universidade do Estado do Rio de Janeiro. Rio de Janeiro, RJ, Brasil.

\section{Jonas L. Gurgel}

Programa de Pós-graduação em Ciências

Cardiovasculares. Universidade Federal Fluminense.

Niterói, RJ, Brasil.

\section{Jonathan Myers}

VA Palo Alto Health Care System. Cardiology Division. Stanford University. Palo Alto, California, United States.

\section{Karynne Grutter}

Laboratório de Anatomia Humana. Universidade Castelo Branco. Rio de Janeiro, RJ, Brasil.

\section{Lenifran Matos-Santos}

Programa de Pós-graduação em Ciências da Atividade Física. Universidade Salgado de Oliveira. Niterói, RJ, Brasil.

\section{Lorena Paes}

Programa de Pós-graduação em Fisiopatologia Clínica e Experimental. Universidade do Estado do Rio de Janeiro. Rio de Janeiro, RJ, Brasil.

\section{Luciane P. da Costa}

Laboratório de Atividade Física e Promoção da Saúde. Instituto de Educação Física e Desportos. Universidade do Estado do Rio de Janeiro. Rio de Janeiro, RJ, Brasil.

\section{Luiz G. Kraemer-Aguiar}

Departamento de Medicina Interna. Faculdade de Ciências Médicas. Universidade do Estado do Rio de Janeiro. Rio de Janeiro, RJ, Brasil.

\section{Matheus R. Hausen}

Laboratório de Atividade Física e Promoção da Saúde. Instituto de Educação Física e Desportos. Universidade do Estado do Rio de Janeiro. Rio de Janeiro, RJ, Brasil.

\section{Paulo T. V. Farinatti}

Laboratório de Atividade Física e Promoção da Saúde. Instituto de Educação Física e Desportos. Universidade do Estado do Rio de Janeiro. Rio de Janeiro, RJ, Brasil.

\section{Rafael A. Montenegro}

Programa de Pós-graduação em Fisiopatologia Clínica e Experimental. Universidade do Estado do Rio de Janeiro. Rio de Janeiro, RJ, Brasil.

\section{Raul A. Freire}

Programa de Pós-graduação em Ciências da Atividade Física. Universidade Salgado de Oliveira. Niterói, RJ, Brasil.

\section{Renato O. Massaferri}

Programa de Pós-graduação em Ciências da Atividade Física. Universidade Salgado de Oliveira. Niterói, RJ, Brasil.

\section{Ricardo B. Oliveira}

Laboratório de Atividade Física e Promoção da Saúde. Instituto de Educação Física e Desportos. Universidade do Estado do Rio de Janeiro. Rio de Janeiro, RJ, Brasil.

\section{Ricardo G. Cordeiro}

Programa de Pós-graduação em Ciências da Atividade Física. Universidade Salgado de Oliveira. Niterói, RJ, Brasil.

\section{Sérgio Machado}

Programa de Pós-graduação em Ciências da Atividade Física. Universidade Salgado de Oliveira. Niterói, RJ, Brasil.

\section{Taciana Pinto}

Laboratório de Atividade Física e Promoção da Saúde. Instituto de Educação Física e Desportos. Universidade do Estado do Rio de Janeiro. Rio de Janeiro, RJ, Brasil.

\section{Tainah P. Lima Monteiro}

Programa de Pós-graduação em Ciências Médicas. Faculdade de Ciências Médicas. Universidade do Estado do Rio de Janeiro. Rio de Janeiro, RJ, Brasil.

\section{Tânia M. P. F. Paschoalino}

Hospital Universitário Antônio Pedro. Universidade Federal Fluminense. Niterói, RJ, Brasil.

\section{Walace D. Monteiro}

Laboratório de Atividade Física e Promoção da Saúde. Instituto de Educação Física e Desportos. Universidade do Estado do Rio de Janeiro. Rio de Janeiro, RJ, Brasil. 